\title{
PRODUTIVIDADE DE GRÃOS EM CULTIVARES DE MILHO TRANSGÊNICAS NA REGIÃO O NORTE/OESTE DO ESTADO DE SÃO PAULO EM 2011/12 E 2012/13
}

ISSUE DOI: $10.3738 /$ nucleus.v0i0.916

FREITAS, Rogério Soares de ${ }^{1,2}$ DUARTE, Aildson Pereira ${ }^{2,3}$

LEÃO, Paulo César da Luz ${ }^{4}$

KASAI, Francisco ${ }^{1,2}$

CAZENTINI-FILHO, Gerson ${ }^{2,5}$

TICELII, Marcelo ${ }^{1,2}$

MINGOTTE, Fábio Luíz Checchio ${ }^{6}$

PESSINATTI, Fernando Augusto ${ }^{6}$

FORNASIERI FILHO, Domingos ${ }^{6}$

BORGES, Wander L. Barbosa ${ }^{1,2}$

VITOR, Leandro Galindo ${ }^{7}$

OLIVEIRA, Antonio Luis de ${ }^{8}$

PONTE, Mayara Silva ${ }^{2,9}$

RESUMO: Foram avaliados 30 híbridos de milho transgênicos na safra de 2011/12 e 30 em 2012/13, em 12 experimentos, sendo comuns nestas avaliações apenas 18 híbridos. Empregou-se o delineamento de blocos ao acaso com três repetições. As parcelas foram constituídas de quatro linhas de 5,0 m de comprimento utilizando-se as duas centrais para as avaliações. O espaçamento entre linhas foi de $0,8 \mathrm{~m}$ e a população inicial de plantas de 62.500 plantas por hectare.De modo geral, a produtividade dos ensaios na safra de 2011/12 foi menor que a verificada em 2012/2013, devido principalmente ao longo período de veranico. Na análise conjunta de dois anos, as cultivares que se destacaram foram DKB310 PRO, AS1598 PRO, 30A37 Hx e AS1581 PRO. Entretanto, diversos outros híbridos produziram mais de $10.000 \mathrm{~kg} \mathrm{ha}^{-1}$ de grãos, indicando que existem várias opções para escolha do híbrido mais adequado a cada sistema de produção e nível de investimento em sementes.

Palavras-chave: Zea mays, sistema de produção, competição de cultivares

SUMMARY: Sixteen transgenic cultivars were evaluated in 12 experiments during 2011/12 and 2012/13 crop seasons. It was used a randomized block design with three replications. The plots consisted of four rows $5.0 \mathrm{~m}$ in length using the two lines for the evaluations. The spacing between rows was $0.8 \mathrm{~m}$ and initial plant population 62,500 plants per hectare. In general, the yield was lower in 2011/12 than 2012/2013, mainly due to the long dry period. In the pooled analysis of two years, cultivars that stood out were DKB310 PRO, PRO AS1598, and AS1581 PRO 30A37 Hx. However, several other hybrids produced over 10,000 kg ha ${ }^{-1}$, indicating several options to choose cultivars suitable for each crop system and level of investment in seeds.

Keywords: Zea mays, crop production system, cultivar competition

\section{INTRODUÇÃO}

A cultura do milho tem recebido altos investimentos em produção de sementes melhoradas, resultando em cultivares com alto potencial produtivo e características agronômicas adequadas aos principais sistemas de produção. A recente liberação do uso de milho $B t$ no Brasil e a rapidez com que esta

\footnotetext{
${ }^{1}$ Polo Regional, Agência Paulista de Tecnologia dos Agronegócios (APTA)

${ }^{2}$ Programa Milho e Sorgo IAC/APTA;

${ }^{3}$ Instituto Agronômico de Campinas

${ }^{4}$ Cati, EDR de Orlândia

${ }^{5}$ Cati, DSMM/NPSFE - Fernandópolis

${ }^{6}$ Unesp, Jaboticabal

${ }^{7}$ Cati, Casa da Agricultura de Ituverava

${ }^{8}$ FAFRAM, Faculdade Dr. Francisco Maeda

${ }^{9}$ FundAg, Fundação de Apoio à Pesquisa Agrícola
} 
tecnologia foi incorporada no sistema de produção do milho demonstram o dinamismo do setor de produção de milho no Brasil. Conforme dados da Associação Paulista de Mudas e Sementes (APPS) citados por Antoniali (2012), a participação de sementes de milho geneticamente modificadas no mercado brasileiro evoluiu de 35,5\% para 78,1\% em curto período de tempo (safra de verão 2009 a safrinha de 2012).

O primeiro evento de milho transgênico resistente a insetos foi aprovado no Brasil em 2007. Esse evento expressa a proteína Cry1Ab de Bacillus thuringiensis Berliner (Bt). Posteriormente, foram aprovados outros eventos que expressam outras proteínas de Bt, tais como Cry1F e Vip3A, bem como os eventos que expressam mais de uma proteína: Cry1A.105/Cry2Ab2, Cry1Ab/Vip3A, Cry1Ab/Cry1F e Cry1A.105/Cry2Ab2/Cry1F (Omoto, 2012). Segundo Cruz (2012), o principal objetivo do milho Bt é reduzir a população da lagarta-do-cartucho, o que de fato vem ocorrendo, apesar de existir diferenças quanto à suscetibilidade entre os vários eventos (GATTI, 2012).

A diferença entre a produtividade média da maioria das lavouras comerciais e a obtida sob condições de alto manejo pode ser atribuída a várias causas, incluindo o uso de genótipos com baixo potencial de produção de grãos e/ou não adaptados à região de cultivo (SANGOI et al. 2007). Em regiões de baixa altitude, como a Norte-Oeste do Estado de São Paulo, que compreende desde áreas próximas de Ribeirão Preto até o extremo Oeste, na divisa do Mato Grosso do Sul, as cultivares devem apresentar tolerância as altas temperaturas noturnas e resistência à ferrugem polissora (Puccinia polysora).

Como o objetivo de indicar as cultivares mais produtivas e adaptadas às condições edafoclimáticas regionais nesta importante região produtora paulista, o Programa Milho e Sorgo IAC/APTA tem realizado uma rede de experimentos continuadamente desde 1996 (DUARTE et al., 1996; DUARTE et al., 1997; DUARTE et al., 1998; CAZENTINI FILHO et al., 2000; FREITAS et al., 2006 e 2009). Os novos lançamentos das empresas são comparados aos padrões comerciais quanto ao desempenho agronômico e à resistência às doenças de ocorrência regional. A partir da 2009/10 foram realizados experimentos com híbridos transgênicos de milho, quando a maioria dos agricultores teve acesso a esta tecnologia. No presente trabalho foram avaliados híbridos de milho transgênicos nas safras 2011/12 e 2012/13.

\section{MATERIAL E MÉTODOS}

Em 2011/2012 e 2012/2013, foram desenvolvidos experimentos em 12 ambientes na região Norte/Oeste do Estado de São Paulo pelo Projeto IAC/APTA/CATI/Empresas. Foram avaliados 30 híbridos em 2011/12 e 33 em 2012/13, sendo apenas 18 comuns nos dois anos, nos municípios de Votuporanga, Pindorama, Adamantina, Riolândia, Colina, Ituverava e Jaboticabal (Quadro 1). Empregouse o delineamento experimental de blocos ao acaso com três repetições. As parcelas foram constituídas de quatro linhas de 5,0 m de comprimento espaçadas de 0,8 m, exceto em Riolândia onde o espaçamento foi de $0,67 \mathrm{~m}$ no verão de 2011 e $0,50 \mathrm{~m}$ em 2012. A população de plantas foi ajustada, após desbaste, entre 10 a 15 dias após a emergência (DAE), para 62.500 plantas por hectare.

O sistema de plantio direto foi utilizado na maioria das áreas, realizando a semeadura após estabilização do período chuvoso, geralmente no mês de novembro (Quadro 1). Na semeadura empregouse pelo menos $300 \mathrm{~kg} \mathrm{ha}^{-1}$ da fórmula NPK 8-28-16 e em cobertura pelo menos $92 \mathrm{~kg} \mathrm{ha}^{-1} \mathrm{de} \mathrm{N}$, quase sempre como sulfato de amônio ou formulação cotendo $\mathrm{N}$ e K (Quadro 1). As sementes foram tratadas com os inseticidas Thiodicarb+Imadacloprid. A cultura foi mantida livre da interferência de plantas infestantes com utilização do herbicida atrazine + tembotrione e/ou capina manual com enxada. Não foi 
realizada aplicação de inseticidas e fungicidas para controle de pragas e doenças respectivamente. As características agronômicas avaliadas nas duas linhas centrais de cada parcela foram: data de florescimento masculino, quando $50 \%$ das plantas estavam com pendão liberando pólen; plantas acamadas e quebradas; altura da planta e da espiga, medida em planta representativa da parcela; massa de grãos e teor de umidade. A produtividade de grãos foi corrigida para 13\% de umidade dos grãos. Para análise dos resultados, procedeu-se à análise de variância e comparação das médias pelo teste de Tukey, a 5\% de probabilidade, usando o programa estatístico do SAS. 
Quadro 1 - Caracterização dos experimentos de milho, desenvolvidos na Região Norte/Oeste do Estado de São Paulo em 2012/13

\begin{tabular}{|c|c|c|c|c|c|c|c|c|c|}
\hline \multirow{3}{*}{ Local } & \multirow{3}{*}{$\begin{array}{c}\text { Responsável } \\
\text { Entidade }\end{array}$} & \multirow{3}{*}{$\begin{array}{c}\text { Altitude } \\
\mathrm{M}\end{array}$} & \multirow{3}{*}{$\begin{array}{l}\text { Solo } \\
\text { Tipo }\end{array}$} & \multirow{3}{*}{$\begin{array}{c}\text { Semeadura } \\
\text { Data }\end{array}$} & \multirow{3}{*}{$\begin{array}{c}\text { Produtividade } \\
\mathrm{kg} \mathrm{ha}^{-1}\end{array}$} & \multicolumn{4}{|c|}{ Adubação } \\
\hline & & & & & & \multicolumn{2}{|c|}{ Semeadura } & \multicolumn{2}{|c|}{ Cobertura $(\mathrm{N})$} \\
\hline & & & & & & $\mathrm{kg} \mathrm{ha}^{-1}$ & NPK & $\mathrm{kg} \mathrm{ha}^{-1(1)}$ & Fonte $^{(2)}$ \\
\hline & & & & & $\underline{2011 / 12}$ & & & & \\
\hline Colina & IAC/APTA & 568 & $\mathrm{LVe}$ & $21 / 11 / 11$ & 12.207 & 350 & $08-28-16$ & $60+60$ & 20-05-20 + S.A. \\
\hline Jaboticabal & Unesp & 595 & LVdf & $18 / 11 / 11$ & 11.751 & 300 & $08-28-16+0,3 \% \mathrm{Zn}$ & $100+100$ & S.A. \\
\hline Riolândia & IAC/APTA e CATI & 420 & LVdf & $24 / 11 / 11$ & 8.788 & 350 & 08-24-12 + micro & $75+40$ & $25-00-25$ + S.A. \\
\hline Adamantina & IAC/APTA & 450 & $\mathrm{LVe}$ & $04 / 11 / 11$ & 8.379 & 650 & 04-14-08 & 120 & S.A. \\
\hline Ituverava & CATI / FAFRAM & 630 & LVdf & $28 / 11 / 11$ & 7.890 & 350 & $08-28-16$ & $32+60$ & 21-00-21 + S.A. \\
\hline Votuporanga & IAC/APTA & 480 & $\mathrm{LVe}$ & $21 / 11 / 11$ & 7.289 & 350 & $08-28-16$ & $60+50$ & 20-00-20 + S.A. \\
\hline & & & & & $\underline{2012 / 13}$ & & & & \\
\hline Jaboticabal & Unesp & 595 & $\mathrm{LVe}$ & $02 / 11 / 12$ & 12.286 & 300 & 04-20-20 + Micro & 210 & S.A. \\
\hline Votuporanga & IAC/APTA & 480 & LVe & $13 / 11 / 12$ & 12.003 & 350 & $08-28-16$ & $64+40$ & 20-00-20 + S.A. \\
\hline Ituverava & CATI / FAFRAM & 631 & LVdf & $21 / 11 / 12$ & 11.947 & 350 & $08-28-16$ & $63+63$ & 21-00-21 + S.A. \\
\hline Riolândia & IAC/APTA e CATI & 420 & LVdf & $03 / 01 / 13$ & 11.227 & 330 & 08-24-12 + Micro & $64+105$ & $20-00-20+30-00-15$ \\
\hline Colina & IAC/APTA & 580 & $\mathrm{LVe}$ & $04 / 12 / 12$ & 10.069 & 320 & $08-28-16$ & $50+50$ & 20-05-20 + S.A. \\
\hline Adamantina & IAC/APTA & 450 & LVe & $30 / 11 / 12$ & 8.522 & 320 & $08-28-16$ & 113 & Ureia \\
\hline
\end{tabular}

${ }^{(1)}$ Quantidade de Nitrogênio. ${ }^{(2)}$ S. A. = Sulfato de Amônio 


\section{RESULTADOS E DISCUSSÃO}

Os ensaios apresentaram coeficiente de variação inferior a $10 \%$, indicando boa precisão experimental. Na safra de 2012/2013, a produtividade média foi de $11.009 \mathrm{~kg} \mathrm{ha}^{-1}$ enquanto que na safra anterior foi de $9.384 \mathrm{~kg} \mathrm{ha}^{-1}$, ou seja, $15 \%$ menor. Considerando o dms do teste Tukey a $5 \%$, as cultivares mais produtivas na safra de 2012/2013 tiveram médias entre $12.249 \mathrm{~kg} / \mathrm{ha}$ a $11.300 \mathrm{~kg} \mathrm{ha}^{-1}$ e na safra de 2011/2012, de 10.484 a $9.400 \mathrm{~kg} \mathrm{ha}^{-1}$ (Quadros 2 e 3). A menor produtividade verificada na ultima safra pode ser explicada, em parte, pelo prolongado período de veranico em alguns locais, principalmente em Votuporanga.

A altura média das plantas (AP) e das espigas (AE) também foram menor em 2011/2012 em relação à safra 2012/2013 (respectivamente de 2,16 m e 2,43 m para AP; 1,18 m e 1,38 m para AE) (Quadros 2 e 3). Na análise conjunta, que inclui os cultivares comuns aos dois anos de avaliação, os híbridos mais produtivas foram DKB 310 PRO, AS 1598 PRO, 30A37 Hx e AS 1581 PRO, que não diferiram pelo teste Tukey a 5\% (Quadro 4) que, juntamente com outros híbridos, tiveram médias superiores a 10 toneladas por hectare. Foi a primeira vez, desde o inícios dos trabalhos na região Oeste, em 1994 (Duarte et al., 2000), que este limite foi ultrapassado em análise conjunta de dois anos, demonstrando a melhoria das cultivares e do manejo cultural.

Acrescenta-se que a incidência de doenças foi muito baixa neste período, evitando possíveis prejuízos na produtividade. Os resultados da avaliação de doenças não foram apresentados porque ocorreu apenas a ferrugem polissora (Puccinia polysora) em dois locais (COLINA; RIOLÂNDIA), com baixa severidade em quase todos os híbridos. Os híbridos que se mostraram mais suscetíveis foram DKB 250 PRO, NS 50 PRO e BX907YG. Como não foram feitas aplicações de fungicidas, as cultivares resistentes apresentaram maior estabilidade produtiva (dados não apresentados).

A produtividade é um critério importante na escolha de um cultivar, mas não é o único. No momento da sua escolha, deve-se considerar também a relação custo/benefício, tendo como base o preço das sementes, a produtividade, a eficiência da tecnologia $B t$ no controle de pragas, a tolerância a doenças de ocorrência regionais e, evidentemente, o nível tecnológico que o produtor utiliza na lavoura.

Quadro 2 - Caracteres agronômicos dos híbridos transgênicos de milho, avaliados em 6 ambientes na Região Norte/Oeste do Estado de São Paulo em 2011/2012

(Continua)

\begin{tabular}{|c|c|c|c|c|c|c|c|c|c|}
\hline \multirow{2}{*}{ Cultivar } & \multicolumn{2}{|c|}{ Altura de } & \multirow{2}{*}{$\begin{array}{l}\text { Rend. } \\
\text { de } \\
\text { espigas }\end{array}$} & \multicolumn{2}{|c|}{ Plantas ${ }^{(2)}$} & \multirow{2}{*}{$\begin{array}{l}\text { Floresc. } \\
\text { masculino }\end{array}$} & \multirow{2}{*}{$\begin{array}{l}\text { Umi- } \\
\text { dade }^{(4)}\end{array}$} & \multirow{2}{*}{ População } & \multirow{2}{*}{$\begin{array}{l}\text { Produti- } \\
\text { Vidade }\end{array}$} \\
\hline & plantas & Espigas & & acam. & queb. & & & & \\
\hline & \multicolumn{2}{|c|}{$\ldots . . \mathrm{cm} \ldots .}$. & & \multicolumn{2}{|c|}{$\ldots . \%$..... } & d.a.s. ${ }^{(3)}$ & $\%$ & Plantas.ha $_{1}^{-}$ & $\mathrm{kg} \cdot \mathrm{ha}^{-1}$ \\
\hline DKB 310 PRO & 219 & 128 & 72 & 0 & 3 & 61 & 18,9 & 63.465 & 10.484 \\
\hline AG 8088 PRO & 204 & 99 & 76 & 2 & 0 & 57 & 18,1 & 63.775 & 10.290 \\
\hline 20A78 Hx & 207 & 115 & 78 & 2 & 2 & 57 & 15,9 & 64.593 & 10.155 \\
\hline 30A37 Hx & 202 & 112 & 75 & 3 & 3 & 57 & 17,5 & 63.370 & 10.027 \\
\hline 2B587 Hx & 204 & 109 & 77 & 2 & 1 & 57 & 18,1 & 62.770 & 9.971 \\
\hline DKB 175 PRO & 226 & 129 & 72 & 3 & 1 & 58 & 18,0 & 62.497 & 9.860 \\
\hline 2B433 Hx & 205 & 113 & 76 & 2 & 4 & 55 & 16,3 & 63.975 & 9.854 \\
\hline DKB 340 PRO & 236 & 130 & 69 & 4 & 1 & 60 & 17,1 & 63.612 & 9.740 \\
\hline P $3862 \mathrm{H}$ & 226 & 124 & 73 & 2 & 2 & 58 & 18,0 & 63.051 & 9.679 \\
\hline 30A95 Hx & 213 & 114 & 76 & 3 & 2 & 57 & 18,1 & 63.051 & 9.646 \\
\hline
\end{tabular}


Quadro 2 - Caracteres agronômicos dos híbridos transgênicos de milho, avaliados em 6 ambientes na Região Norte/Oeste do Estado de São Paulo em 2011/2012

(Conclusão)

\begin{tabular}{lccccccccc} 
AG 8061 PRO & 202 & 109 & 74 & 1 & 1 & 58 & 18,0 & 64.773 & 9.612 \\
AS 1581 PRO & 227 & 124 & 72 & 3 & 2 & 61 & 17,8 & 62.292 & 9.584 \\
DKB 390 PRO & 209 & 115 & 74 & 9 & 0 & 57 & 15,8 & 64.317 & 9.582 \\
30A16 Hx & 221 & 115 & 71 & 2 & 1 & 59 & 20,6 & 62.411 & 9.567 \\
AS 1598 PRO & 224 & 122 & 72 & 3 & 2 & 60 & 18,0 & 62.840 & 9.546 \\
2B707 Hx & 215 & 115 & 73 & 3 & 1 & 59 & 18,4 & 63.119 & 9.537 \\
AG 8676 PRO & 230 & 127 & 71 & 2 & 1 & 60 & 17,6 & 63.713 & 9.484 \\
AS 1625 PRO & 217 & 118 & 71 & 6 & 3 & 61 & 15,6 & 62.783 & 9.467 \\
AG 7098 PRO & 214 & 125 & 74 & 0 & 2 & 60 & 18,8 & 62.604 & 9.453 \\
GNZ 9626 PRO & 220 & 122 & 70 & 2 & 2 & 58 & 18,2 & 62.506 & 9.446 \\
2B604 Hx & 217 & 112 & 72 & 7 & 1 & 57 & 18,8 & 64.629 & 9.227 \\
2B710 Hx & 205 & 105 & 74 & 1 & 1 & 56 & 19,9 & 63.192 & 9.193 \\
BG 7049 H & 223 & 121 & 73 & 8 & 1 & 58 & 17,6 & 62.445 & 8.836 \\
30F35 H & 229 & 119 & 73 & 2 & 0 & 59 & 17,6 & 63.226 & 8.728 \\
AS 3421 YG & 213 & 116 & 71 & 4 & 3 & 60 & 18,4 & 62.917 & 8.704 \\
SG 6030 YG & 220 & 124 & 73 & 12 & 3 & 57 & 17,2 & 63.906 & 8.701 \\
Truck VIP & 212 & 120 & 74 & 4 & 1 & 57 & 17,1 & 63.180 & 8.403 \\
GNZ 9688 PRO & 218 & 123 & 70 & 2 & 2 & 57 & 16,2 & 63.088 & 8.298 \\
Status VIP & 215 & 121 & 72 & 2 & 2 & 57 & 17,0 & 64.678 & 8.236 \\
DKB 350 YG & 200 & 110 & 73 & 6 & 3 & 56 & 16,4 & 63.333 & 8.208 \\
\hline Média & 216 & 118 & 73 & 3 & 2 & 58 & 17,7 & 63.337 & 9.384 \\
CV (\%) & 5,1 & 8,3 & 4,8 & - & - & - & 3,3 & 5,0 & 10,1 \\
Dms ${ }^{(5)}$ & 12 & 11 & 4 & - & - & - & 0,9 & 3.442 & 1.030 \\
\hline
\end{tabular}

${ }^{(1)}$ Locais: Adamantina, Cardoso, Colina, Ituverava, Jaboticabal e Votuporanga. ${ }^{(2)}$ Plantas acamadas e quebradas. ${ }^{(3)}$ Dias após semeadura, exceto em Jaboticabal e Ituverava. ${ }^{(4)}$ Teor de água nos grãos na colheita, em Adamantina, Cardoso e Votuporanga; ${ }^{(5)}$ Diferença mínima significativa, a $5 \%$ de probabilidade, pelo teste Tukey.

Quadro 3 - Caracteres agronômicos dos híbridos transgênicos de milho, avaliados em 6 ambientes na Região Norte/Oeste do Estado de São Paulo em 2012/2013 ${ }^{(1)}$.

(Continua)

\begin{tabular}{|c|c|c|c|c|c|c|c|c|c|}
\hline \multirow{2}{*}{ Cultivar } & \multicolumn{2}{|c|}{ Altura de } & \multirow{2}{*}{$\begin{array}{l}\text { Rend. } \\
\text { de } \\
\text { espigas }\end{array}$} & \multicolumn{2}{|c|}{ Plantas ${ }^{(2)}$} & \multirow{2}{*}{$\begin{array}{l}\text { Floresc. } \\
\text { masculino }\end{array}$} & \multirow{2}{*}{$\begin{array}{l}\text { Umi- } \\
\text { dade }^{(4)}\end{array}$} & \multirow{2}{*}{ População } & \multirow{2}{*}{$\begin{array}{l}\text { Produti- } \\
\text { Vidade }\end{array}$} \\
\hline & plantas & espigas & & acam. & queb. & & & & \\
\hline & \multicolumn{2}{|c|}{$\ldots . . . \mathrm{cm} \ldots .}$. & ......... & $\% \ldots \ldots$ & $\ldots$. & d.a.s. ${ }^{(3)}$ & $\%$ & plantas.ha $^{-1}$ & kg.ha ${ }^{-1}$ \\
\hline AS 1598 PRO & 253 & 147 & 77 & 1 & 1 & 57 & 21,7 & 62.296 & 12.249 \\
\hline DKB 310 PRO & 248 & 149 & 77 & 1 & 1 & 58 & 21,0 & 62.528 & 11.994 \\
\hline AS 1633 PRO & 252 & 143 & 75 & 0 & 0 & 53 & 19,4 & 62.819 & 11.965 \\
\hline $\begin{array}{l}\text { ADV } 9434 \\
\text { PRO }\end{array}$ & 262 & 155 & 78 & 1 & 1 & 56 & 17,8 & 62.838 & 11.848 \\
\hline AS 1581 PRO & 251 & 147 & 76 & 0 & 1 & 57 & 21,7 & 62.947 & 11.828 \\
\hline 30A37 Hx & 225 & 128 & 80 & 0 & 4 & 53 & 17,9 & 62.775 & 11.462 \\
\hline 30A16 Hx & 244 & 129 & 76 & 0 & 0 & 56 & 22,4 & 62.902 & 11.414 \\
\hline AS 1625 PRO & 254 & 147 & 76 & 1 & 2 & 57 & 19,9 & 62.726 & 11.393 \\
\hline BG $7032 \mathrm{H}$ & 264 & 155 & 75 & 1 & 0 & 57 & 20,3 & 62.471 & 11.378 \\
\hline DKB 340 PRO & 267 & 157 & 74 & 1 & 1 & 57 & 19,2 & 62.994 & 11.325 \\
\hline P $3862 \mathrm{H}$ & 253 & 144 & 75 & 1 & 1 & 56 & 20,7 & 62.042 & 11.317 \\
\hline AG 7098 PRO & 238 & 141 & 77 & 0 & 0 & 56 & 20,4 & 62.295 & 11.202 \\
\hline $\begin{array}{l}\text { DKB } 390 \\
\text { PRO2 }\end{array}$ & 241 & 135 & 78 & 0 & 2 & 54 & 16,8 & 63.235 & 11.175 \\
\hline
\end{tabular}

Nucleus, Edição Especial, 2013 
Quadro 3 - Caracteres agronômicos dos híbridos transgênicos de milho, avaliados em 6 ambientes na Região Norte/Oeste do Estado de São Paulo em 2012/2013 ${ }^{(1)}$.

(Conclusão)

\begin{tabular}{lccccccccc} 
2B604 Hx & 244 & 132 & 76 & 0 & 2 & 54 & 18,9 & 63.472 & 11.151 \\
AS 1596 PRO2 & 244 & 141 & 76 & 1 & 1 & 57 & 18,3 & 62.800 & 11.048 \\
DKB 175 PRO & 256 & 152 & 76 & 1 & 2 & 56 & 18,3 & 62.627 & 11.029 \\
30F35 H & 253 & 135 & 76 & 1 & 1 & 55 & 19,0 & 62.722 & 11.026 \\
P 3646 H & 238 & 129 & 77 & 1 & 7 & 54 & 17,6 & 61.809 & 10.974 \\
Truck VIP & 239 & 134 & 78 & 0 & 3 & 54 & 19,2 & 62.300 & 10.960 \\
2B512 Hx & 227 & 127 & 79 & 0 & 4 & 53 & 17,7 & 62.566 & 10.953 \\
AG 8088 PRO2 & 221 & 116 & 77 & 2 & 1 & 54 & 18,1 & 63.251 & 10.952 \\
20A78 Hx & 229 & 132 & 79 & 0 & 3 & 53 & 17,8 & 63.099 & 10.918 \\
LG 6036 PRO & 250 & 143 & 75 & 0 & 3 & 58 & 18,9 & 61.886 & 10.885 \\
AG 8676 PRO & 248 & 147 & 74 & 0 & 1 & 57 & 18,1 & 62.157 & 10.816 \\
AG 8061 PRO & 227 & 129 & 76 & 2 & 1 & 55 & 19,1 & 62.609 & 10.680 \\
2B433 Hx & 226 & 125 & 79 & 0 & 3 & 52 & 17,5 & 62.883 & 10.662 \\
AG 5055 PRO & 244 & 141 & 75 & 0 & 2 & 57 & 19,0 & 62.456 & 10.659 \\
Status VIP & 241 & 140 & 77 & 0 & 6 & 54 & 20,2 & 62.877 & 10.555 \\
DKB 250 PRO & 246 & 134 & 82 & 0 & 6 & 54 & 15,9 & 62.061 & 10.462 \\
NS 50 PRO & 243 & 128 & 80 & 0 & 3 & 52 & 16,1 & 61.903 & 10.164 \\
SG 6030 YG & 252 & 152 & 77 & 5 & 2 & 53 & 17,9 & 62.926 & 10.087 \\
DKB 350 PRO & 223 & 122 & 75 & 0 & 4 & 53 & 18,7 & 63.047 & 9.515 \\
BX 907 YG & 224 & 112 & 81 & 1 & 7 & 51 & 14,7 & 62.101 & 9.252 \\
\hline Média & 243 & 138 & 77 & 1 & 2 & 55 & 18,8 & 62.619 & 11.009 \\
CV (\%) & 4,4 & 6,7 & 2,2 & - & - & - & 5,3 & 3,8 & 7,5 \\
Dms & 12 & 10 & 2 & - & - & - & 1,6 & 2.615 & 910 \\
\hline
\end{tabular}

${ }^{\left({ }^{(1)}\right.}$ Locais: Adamantina, Colina, Ituverava, Jaboticabal, Riolândia e Votuporanga. ${ }^{(2)}$ Plantas acamadas e quebradas.

${ }^{(3)}$ Dias após semeadura, exceto em Jaboticabal e Ituverava. ${ }^{(4)}$ Teor de água nos grãos na colheita, em Adamantina, Colina e Riolândia; ${ }^{(5)}$ Diferença mínima significativa, a 5\% de probabilidade, pelo teste Tukey.

Quadro 4 - Caracteres agronômicos dos híbridos transgênicos de milho, avaliados em 12 ambientes na Região Norte/Oeste do Estado de São Paulo em 2011/2012 e 2012/2013

(Continua)

\begin{tabular}{|c|c|c|c|c|c|c|c|c|c|c|c|}
\hline \multirow{2}{*}{ Cultivar } & \multirow{2}{*}{$\operatorname{Tipo}^{(2)}$} & \multicolumn{2}{|c|}{ Altura de } & \multirow{2}{*}{$\begin{array}{l}\text { Rend. de } \\
\text { espigas }^{(3)}\end{array}$} & \multicolumn{2}{|c|}{ Plantas } & \multirow{2}{*}{ Floresc. } & \multirow{2}{*}{ Umid. $^{(5)}$} & \multirow{2}{*}{ População } & \multirow{2}{*}{\multicolumn{2}{|c|}{$\begin{array}{l}\text { Produti- } \\
\text { vidade }^{(6)}\end{array}$}} \\
\hline & & Planta & Espigas & & Acam. & Queb. & & & & & \\
\hline & & \multicolumn{2}{|c|}{$\ldots . \mathrm{cm} \ldots}$. & \multicolumn{3}{|c|}{$\ldots \ldots . . \%$} & d.a.s. ${ }^{(4)}$ & $\%$ & plantas ha $^{-1}$ & $\mathrm{~kg} \mathrm{ha}^{-1}$ & \\
\hline DKB 310 PRO & HS & 234 & 138 & 74 & 0 & 2 & 59 & 20,0 & 62.997 & 11.239 & $\mathrm{a}$ \\
\hline AS 1598 PRO & HS & 239 & 134 & 74 & 2 & 1 & 58 & 19,8 & 62.568 & 10.898 & $a b$ \\
\hline 30A37 Hx & HS & 213 & 120 & 78 & 2 & 4 & 55 & 17,7 & 63.073 & 10.745 & $a-c$ \\
\hline AS 1581 PRO & HS & 239 & 135 & 74 & 2 & 2 & 59 & 19,8 & 62.619 & 10.706 & $a-c$ \\
\hline 20A78 Hx & HT & 218 & 123 & 79 & 1 & 2 & 55 & 16,9 & 63.846 & 10.537 & $\mathrm{bc}$ \\
\hline DKB 340 PRO & HS & 252 & 144 & 71 & 3 & 1 & 58 & 18,2 & 63.303 & 10.532 & $b-c$ \\
\hline P $3862 \mathrm{H}$ & HS & 239 & 134 & 74 & 1 & 2 & 57 & 19,3 & 62.547 & 10.498 & $b-c$ \\
\hline 30A16 Hx & HS & 233 & 122 & 74 & 1 & 1 & 57 & 21,5 & 62.657 & 10.491 & $b-c$ \\
\hline AS 1625 PRO & HS & 235 & 133 & 73 & 3 & 3 & 59 & 17,8 & 62.755 & 10.430 & $b-c$ \\
\hline AG 7098 PRO & HS & 226 & 133 & 76 & 0 & 1 & 58 & 19,6 & 62.450 & 10.328 & $b-e$ \\
\hline 2B433 Hx & HT & 216 & 119 & 78 & 1 & 3 & 53 & 16,9 & 63.429 & 10.258 & $b-e$ \\
\hline
\end{tabular}


Quadro 4 - Caracteres agronômicos dos híbridos transgênicos de milho, avaliados em 12 ambientes na Região Norte/Oeste do Estado de São Paulo em 2011/2012 e 2012/2013

(Conclusão)

\begin{tabular}{|c|c|c|c|c|c|c|c|c|c|c|c|}
\hline 2B604 Hx & $\mathrm{HSm}$ & 231 & 122 & 74 & 4 & 2 & 55 & 18,9 & 64.051 & 10.189 & c-e \\
\hline AG 8676 PRO & HS & 239 & 137 & 73 & 1 & 1 & 58 & 17,9 & 62.935 & 10.150 & c-e \\
\hline AG 8061 PRO & HS & 215 & 119 & 75 & 2 & 1 & 56 & 18,5 & 63.691 & 10.146 & c-e \\
\hline $30 F 35 \mathrm{H}$ & HS & 241 & 127 & 75 & 2 & 1 & 57 & 18,3 & 62.974 & 9.877 & $d-f$ \\
\hline Truck VIP & HS & 225 & 127 & 76 & 2 & 2 & 56 & 18,1 & 62.740 & 9.682 & ef \\
\hline Status VIP & HS & 228 & 131 & 74 & 1 & 4 & 55 & 18,6 & 63.778 & 9.395 & $\mathrm{f}$ \\
\hline SG 6030 YG & HS & 236 & 138 & 75 & 9 & 2 & 55 & 17,5 & 63.416 & 9.394 & $\mathrm{f}$ \\
\hline Média & & 231 & 130 & 75 & 2 & 2 & 57 & 18,6 & 63.101 & 10.305 & \\
\hline $\mathrm{CV}(\%)$ & & 4,8 & 7,2 & 3,6 & - & - & - & 4,5 & 4,4 & 8,9 & \\
\hline $\mathrm{dms}^{(7)}$ & & 8 & 7 & 2 & - & - & - & 1,0 & 2.007 & 656 & \\
\hline
\end{tabular}

\section{REFERÊNCIAS}

CAZENTINI FILHO, G.et al. Fatores bióticos e abióticos em cultivares de milho e estratificação ambiental; Avaliação IAC/CATI/Empresas 1999/2000. Campinas, IAC, 2000. p. 47-56 (Boletim Científico 05)

CONAB - Companhia Nacional de Abastecimento. Disponível em: <

http://www.conab.gov.br/conteudos.php?a=1252\&t=2\&Pagina_objcmsconteudos=2\#A_objcmsconteudos >. Acesso em 01 jun. 2013.

CRUZ, I. Manejo de pragas não alvo de milho $\mathrm{B} t$ incidentes na parte aérea da planta. In: PATERNIANI, M.E.G.Z.; DUARTE, A.; TSUNECHIRO, A. Diversidade e inovações na cadeia produtiva de milho e sorgo na era dos transgênicos. Campinas, 2012. p. 321-339.

DUARTE, A.P. ; PATERNIANI, M.E.A.G.Z. Avaliação de Cultivares de Milho no Estado de São Paulo. In: DUARTE, A.P.; PATERNIANI, M.E.A.G.Z. Cultivares de milho no Estado de São Paulo: Resultados das avaliações regionais - IAC/CATI/Empresas - 1996/1997. Campinas: Instituto Agronômico, 1997. p. 9-96. (Documento IAC, 58)

DUARTE, A.P.et al. Avaliação de cultivares de milho no Estado de São Paulo. In: DUARTE, A.P.; PATERNIANI, M.E.A.G.Z. Caracterização edafoclimática e avaliação de cultivares de milho no Estado de São Paulo. Campinas: IAC, 1996. p. 31-78. (Documento IAC, 56)

DUARTE, A.P.et al. Milho Safrinha: técnicas para o cultivo no Estado de São Paulo. CATI, Campinas, 2000. 16p. (Documento técnico, 113)

FREITAS, R. S.et al. Avaliação de cultivares de milho na Região Oeste do Estado de São Paulo em 2004/2005 e 2005/2006. In: CONGRESSO NACIONAL DE MILHO E SORGO, 26., 2006, Belo Horizonte. Resumos... Belo Horizonte: ABMS, 2006. p. 217. 
FREITAS, R.S.et al. Avaliação de cultivares de milho na região noroeste do Estado de São Paulo em 2007/2008 e 2008/2009. Nucleus, Edição Especial, Ituverava, 2009, p.53-59.

GATTI, J.H.et al. Eficiência de diferentes tecnologias $\mathrm{B} t$ no Controle de pragas na safrinha: Controle da Lagarta-do-Cartucho. In: CONGRESSO NACIONAL DE MILHO E SORGO, 29., 2012, Águas de Lindoia. Resumos... Águas de Lindoia: ABMS, 2012, p.973-978. CD-ROM.

OMOTO, C. et al. Estratégias de Manejo da Resistência e Importância das Áreas de Refúgio para a Tecnologia Bt. In: PATERNIANI, M.E.G.Z.; DUARTE, A.; TSUNECHIRO, A. Diversidade e Inovações na Cadeia Produtiva de Milho e Sorgo na Era dos Transgênicos. Campinas, 2012. p. 303314.

SANGOI, L. et al. Desenvolvimento e exigências climáticas da planta de milho para altos rendimentos. Lages, SC: 95p, 2007. 
